\title{
L-dopa responsiveness in early Parkinson's disease is associated with the rate of motor progression.
}

Naveed MALEK, MD [1], Sofia KANAVOU, MSc [2], Michael A LAWTON, MPhil [2], Vanessa PITZ MRes [3], Katherine A GROSSET, MD [3], Nin BAJAJ, PhD [4], Roger A BARKER, PhD [5], Yoav BEN-SHLOMO MD, PhD [2], David J BURN, MD [6], Tom FOLTYNIE, PhD [7], John HARDY, PhD [8], Nigel M WILLIAMS, PhD [9], Nicholas WOOD, PhD [10], Huw R MORRIS PhD [11], Donald G GROSSET, MD [3], on behalf of the PRoBaND clinical consortium

1. Department of Neurology, East Suffolk and North Essex NHS Foundation Trust, Ipswich; 2. School of Social and Community Medicine, University of Bristol, Bristol; 3. Institute of Neurological Sciences, Queen Elizabeth University Hospital, Glasgow; 4. Department of Neurology, University of Nottingham; 5. Department of Clinical Neurosciences, John van Geest Centre for Brain Repair, Cambridge; 6. Faculty of Medical Sciences, University of Newcastle, Newcastle upon Tyne; 7. Department of Clinical and Movement Neurosciences, UCL Institute of Neurology, London; 8. Reta Lila Weston Laboratories, Department of Molecular Neuroscience, UCL Institute of Neurology, London; 9. Institute of Psychological Medicine and Clinical Neurosciences, MRC Centre for Neuropsychiatric Genetics and Genomics, Cardiff University, Cardiff; 10. Department of Molecular Neuroscience, UCL Institute of Neurology, London; 11. Department of Clinical Neuroscience, UCL Institute of Neurology, London.

Corresponding author: Naveed Malek, Department of Neurology, East Suffolk and North Essex NHS Foundation Trust, Ipswich, IP4 5PD. Email: nmalek@nhs.net

Key words: Parkinson's disease, L-Dopa, therapy 


\section{Abstract}

Background: L-dopa responsiveness in Parkinson's disease (PD) varies, but the clinical correlates and significance of this are ill-defined.

Methods: Patients were assessed before and after their usual morning L-dopa dose, using the MDS Unified PD Rating Scale Part 3 (MDS UPDRS 3), and rated as definite responders ( $\geq 24.5 \%$ improvement) or limited responders $(<24.5 \%)$.

Results: 1007 cases, mean age 66.1 years (SD 9.1) at diagnosis, were assessed 3.4 (SD 0.9) years after diagnosis. The L-dopa response was definite in 614 cases (61.0\%), median reduction in MDS UPDRS 3 scores was 42.0\%, (IQR 33.3, 53.1), and was limited in 393 cases (39.0\%), median reduction in MDS UPDRS 3 scores $11.5 \%$ (IQR 4.3, 18.2). Definite responders were younger (66.3 years at study entry, SD 9.3) than limited responders (69.2 years, SD 8.4, $\mathrm{p}<0.001)$. The MDS UPDRS 3 score at study entry in definite responders (21.0, SD 10.5) was significantly lower than in limited responders (24.7, SD 13.4, p<0.001). The MDS UPDRS 3 increase over 18 months was less in definite responders at 3.0 (SD 10.4), compared to limited responders $(6.4, S D 11.0, p<0.001)$. The levodopa equivalent daily dose (LEDD) was not significantly different at study entry (definite responders $317 \mathrm{mg}$, SD 199, vs limited responders 305mg, SD 191, $\mathrm{p}=0.53$ ). However, LEDD was significantly higher at the time of the L-dopa challenge test in definite responders $(541 \mathrm{mg}, \mathrm{SD}$ 293) compared to limited responders (485mg, SD 215, $p=0.01$ ). Responsiveness to L-dopa was unaffected by the challenge test dose $(p=0.54)$.

Conclusions: The main determinants of variation in the L-dopa response in early PD are age and motor severity. A limited L-dopa response is associated with faster motor progression. 


\section{Introduction}

The phenotypic heterogeneity of Parkinson's disease (PD) is well recognized[1], and includes variability in L-dopa responsiveness[2, 3]. While an excellent [4] or clear and dramatic [5] response to L-dopa is a supportive feature in the diagnostic criteria for idiopathic PD, a less marked response does not rule out the diagnosis of PD [4, 5]. A number of studies have shown variation in the response to either an acute Ldopa challenge dose $[6,7,8]$, or chronic L-dopa therapy $[2,4,6,8]$ both in clinically diagnosed PD [2, 4, 6, 8], and in pathologically confirmed cases [4]. However, the clinical correlates and significance of this variation in response are not clearly defined. We wished to explore in detail, in a large cohort of recently diagnosed patients, which clinical characteristics are associated with the level of motor responsiveness to L-dopa. We hypothesised that a limited motor response to L-dopa was a likely contributor to variation in the motor progression rate, which could be measured by prospective observation. We also set out to examine whether comorbidities (e.g. cerebrovascular disease) influenced the L-dopa motor response.

\section{Methods}

Tracking Parkinson's is a prospective observational multicentre study that has recruited from 72 centres in the United Kingdom (UK). Patients were recruited with a clinical diagnosis of PD made by a clinician if they fulfilled UK Brain Bank criteria [4]. This was supported by structural and/or functional neuroimaging when the diagnosis was not firmly established clinically. Both drug-naïve and treated patients, aged 18 to 90 years were eligible. Recent onset cases (diagnosed in the preceding 3.5 years), were recruited between February 2012 and May 2014. Patients were not enrolled if they had severe comorbid illness, other degenerative forms of parkinsonism such as multiple system atrophy (MSA), or parkinsonism attributable to significant cerebrovascular disease. Patients with drug-induced parkinsonism were excluded, but drug-unmasked PD was allowed if justified by abnormal functional dopaminergic imaging. Patients whose diagnosis was later revised from PD during regular followup were excluded from any analysis.

Any features (signs, symptoms, progression, medication response) that were possibly unusual or atypical for PD (and might be early pointers to atypical 
parkinsonian disorders such as MSA) were noted at recruitment, and during followup. These cases were included in the main analysis, but excluded from the subanalysis. The study was approved by the West of Scotland Ethics Committee, written informed consent was obtained from patients, and procedures followed the Declaration of Helsinki. The study was supported by funding from Parkinson's UK. The following features were collected in all patients: demographic characteristics, diagnostic features at presentation, medication history, Movement Disorder Society Unified Parkinson's Disease Rating Scale (MDS UPDRS) scores [9] including part 3 scores 'on' or 'off' medication ('off' being defined operationally as below), non-motor symptom severity scale (NMSS) [10], Rapid eye movement sleep (REM) sleep behaviour disorder scale [11] and the Scale for Outcomes in Parkinson's disease for Autonomic Symptoms (SCOPA-AUT) [12]. Motor phenoytpe was categorized according to defined methods [13]. Montreal cognitive assessment (MoCA) test scores were adjusted for years of education. Hyposmia was defined using a Sniffin' Stick score of less than or equal to the $10^{\text {th }}$ centile, or at or below the $15^{\text {th }}$ centile on the University of Pennsylvania Smell Test score (British version), corrected for age and sex [14]. Presence of low mood and depression was assessed using the Leeds Anxiety and Depression Scale (LADS) with a cut-off score >6 [15]. L-dopa equivalent daily dose (LEDD) was calculated using an established formula [16].

Vascular risk was calculated by standard methods, using the QRISK2 algorithm, as previously reported [17]. Rheumatoid arthritis was included as a vascular risk equivalent, as this confers an increased vascular risk [18].

At the 2 year visit an L-dopa challenge test was carried out in patients prescribed Ldopa based therapy. Patients were asked to omit their usual morning antiparkinsonian medication. Those co-prescribed a dopamine agonist omitted this from the night before the L-dopa challenge test (for immediate release preparations) and the day before (for once-daily preparations). Assessments were undertaken in an 'off' state and was repeated 1 hour after the patient's usual morning dose of L-dopa, expressed as the dose of L-dopa in $\mathrm{mg}$ (the L-dopa:decarboxylase ratio was 4:1). The change in MDS UDPRS 3 was dichotomised around a $24.5 \%$ improvement, which is equivalent to the $30 \%$ change in the UPDRS 3 score [7] and defined as 'definite' when improvement was $\geq 24.5 \%$, and 'limited' when $<24.5 \%$. 
Statistical analysis

A logistic regression model was used with response to L-dopa challenge test as the outcome, and the 'limited' group as the baseline category. Odds ratios, 95\% confidence intervals and $p$-values were calculated, before and after adjustment for age, gender and disease duration. Sensitivity analysis was implemented for missing values in the outcome and clinical variables. MoCA, UPDRS 3, SCOPA-AUT, NMSS, olfaction tests scores, and motor responsiveness to L-dopa, were calculated based on expected scores, when at least $80 \%$ of the questions were answered in each scale. Any remaining missing values were then imputed 10 times, using the multiple imputation chained equations approach. Odds ratios and $p$-values were calculated in the logistic regression models using Rubin's rules. Statistical analysis was conducted at $5 \%$ significance level, using STATA/MP software version 15.1 .

\section{Results}

There were 2006 patients recruited, of whom 37 were excluded either due to disease duration over 3.5 years $(n=6)$ or change in diagnosis after recruitment $(n=31)$. A further 429 did not have data for the 2-year visit, leaving 1540 cases, of whom 228 were not prescribed L-dopa at the time of the visit, and 305 in whom data collection was incomplete. Accordingly, complete data were available for the L-dopa challenge in 1007 cases (Table 1).

These cases had a mean age of 67.5 years (SD 9.1) at study entry, and the L-dopa response was assessed on average 3.4 years (SD 0.9) after diagnosis. A definite Ldopa response was seen in 614 cases (61.0\%). The mean change in MDS UPDRS 3 from pre to post challenge L-dopa dose was 42.0\% (IQR 33.3, 53.1) in those with a definite response, versus $11.5 \%$ (IQR 4.3, 18.2) in those with a limited response. Patients who showed a definite L-dopa response were younger (66.3 years, SD 9.3) compared to those with a limited response (69.2 years, SD 8.4, $\mathrm{p}<0.001)$. Those with a definite response had a lower MDS UPDRS 3 score at study entry (21.0, SD 10.5) than those with a limited response (24.7, SD 13.4, p<0.001) (Figure 2). Further, those with a definite response showed a smaller increment in MDS UPDRS 3 scores over the next 18 months (increase of 3.0 points, SD 10.4) compared to an increase of 6.4 points (SD 11.0) in those with a limited response, $p<0.001$. The demographic 
and phenotypic characteristics of the cases who undertook the L-dopa challenge test are shown in Table 1. Using $24.5 \%$ as the cut-off for defining definite responders and limited responders showed a bell-shaped curve (Supplementary Figure 1). The LEDD was not significantly different at study entry comparing those with a definite response (317mg, SD 199) to those with a limited response (305mg, SD 191, $p=0.53)$. However, the LEDD at the time of the challenge test was higher in those with a definite response (541mg, SD 293) compared to those with a limited response (485mg, SD 215, $\mathrm{p}=0.01$ ). The proportion of cases with an unequivocal motor response was higher in definite responders (25.0\%) compared to limited responders $(17.8 \%, p=0.0106)$. More patients classified as definite responders were prescribed a monoamine oxidase type B inhibitor (34.0\%) than those classified as limited responders $(27.0 \%, p=0.0213)$. The increase in the MDS UPDRS 3 scores in the 283 cases that were L-dopa naïve at study entry, from baseline to the 'off' score prior to the challenge test, did not differ significantly when comparing definite responders to limited responders $(p=0.8$, Table 1$)$. There were no statistical differences in disease duration, sex, motor subtype, cognitive scores, non-motor scores, hyposmia, presence of a rapid eye movement behaviour disorder, constipation, depression or autonomic scores between those with a definite versus a limited L-dopa response. Considering comorbid vascular disease and vascular risk factors, only $49.4 \%$ of the 89 patients with Type 2 diabetes showed a definite L-dopa response. In patients with a prior history of vascular disease $(n=149) 53.7 \%$ showed a definite $L$-dopa response. Similarly, in patients with high vascular QRISK2 scores $(n=345), 56.2 \%$ had a definite response to L-dopa, compared to $61.6 \%$ with medium vascular risk scores $(n=295)$, and $72.6 \%$ with low vascular risk scores $(n=215)$. However, these observations were not statistically significant after adjusting for age, sex, and disease duration. There were no significant differences between definite and limited responders for any of the following: prior vascular diagnosis (cardiac or cerebral), rheumatoid arthritis, body mass index, the presence of orthostatic hypotension, or smoking status.

The mean dose of L-dopa used in the acute challenge test was not significantly different between definite and limited responders, whether considering actual dose, or dose per kilogram body weight (Table 2). There was also no significant difference according to fulfilment of MDS diagnostic criteria for PD, comparing definite and limited L-dopa responders (Table 2). 
In the sensitivity analysis all 1312 subjects on L-dopa at the 2-year visit were assessed (Figure 1). Missing data were relatively low ( $<10 \%)$, except in SCOPAAUT (38.5\%), NMSS (22.3\%) and L-dopa motor response (23.3\%). Results did not differ, in any of the key findings, in the sensitivity analysis compared to the main analysis (results not shown).

In the sub-analysis in 949 cases without any unusual or atypical features $(94.2 \%$ of the 1007 cases) the significant findings of the relationship between L-dopa response remained: age $(p<0.001)$, motor severity $(p<0.001)$, and motor progression $(p=0.001)$.

\section{Discussion}

Our main finding is that, in a large cohort of prospectively recruited recent onset PD patients, there is a substantial variation in the degree of motor response to L-dopa. These results are consistent with variation in L-dopa responsiveness in clinical trials and pathological case series [2-4]. In the ELLDOPA study in 260 L-dopa treated patients, the average improvement in UPDRS 3 was $27.4 \%$ (SD 30.6) at 9 weeks, and $26.2 \%$ (SD 36.4) at 24 weeks [2, 3]. Because of the known differences between the UPDRS 3 and MDS UPDRS Part 3 scores [7] the comparable percentage improvements in the ELLDOPA study using the MDS UPDRS 3 would give improvements of $33.6 \%$ (SD 37.5) at 9 weeks and 32.1\% (SD 44.6) at 24 weeks. Variation in the degree of L-dopa response has also been reported in pathologically confirmed PD [4]. In 69 of 76 confirmed PD cases in whom the L-dopa response was available, it was graded as definite in $29 \%$, good in $39 \%$, limited in $13 \%$, and nil-topoor in $4 \%$ [4]. Taken together, we can conclude that the degree of motor improvement in response to L-dopa is subject to significant variation, although the reasons for this are unclear. We therefore sought to better define what factors may be important in determining the level of response.

First we examined demographic and disease related features and found that older patients showed a less robust response to L-dopa, which has been observed previously [19]. However, we did not confirm the previously reported associations of male gender and postural instability with lower L-dopa response rates [2]. We did 
however identify a significant relationship between L-dopa responsiveness and baseline motor scores, as well as rates of motor progression over an 18-month period. These findings are of clinical significance: patients with lower responsiveness to L-dopa have higher motor scores, and faster motor progression. A relationship between increasing age and progression of disability has been observed previously, and attributed in part to L-dopa non-responsive motor symptoms [20]. Further, we observed that more definite responders were prescribed monoamine oxidase type $B$ inhibitors, compared to limited responders, which is in keeping with mechanisms and responses for this drug class [21].

Secondly, given our previous finding of higher motor scores and more cognitive problems in patients with vascular disease, or increased vascular risk factors [17], and the known association of vascular disease with age, we examined for any relationship between vascular comorbidity and L-dopa response in the present study. While we found that both diabetes and higher vascular risk scores were associated with lower L-dopa responsiveness, these observations were driven by patient age, so we can conclude that vascular comorbidity is not an independent driver of an impaired L-dopa response.

There are several other potential explanations for variation in L-dopa responsiveness. Diagnostic inaccuracy is known to occur in cases with an early clinical diagnosis of PD [4, 5], and a poor or absent L-dopa responses in such erroneously diagnosed cases would affect the response levels reported. We controlled for this confounding factor in 2 ways, firstly by prospective collection of structured information of individual clinical features that might be atypical for PD, with analysis after excluding these cases, and secondly by considering the L-dopa response in relation to fulfilment of MDS diagnostic criteria [22]. We did not find major differences using either approach, compared to the main analysis: an additional $1.3 \%$ of cases had a definite response after excluding possible atypical features, while there was no indication of differences in L-dopa response according to categorisation by MDS criteria into 'established PD' or 'probable PD', versus those not fulfilling PD criteria. Taken together, it appears that diagnostic error is not a major contributor to the variation observed in L-dopa responsiveness in our study. 
The dose of L-dopa used to assess responsiveness is clearly important and is another source of variation in our study and others. We used the patient's standard morning L-dopa dose in the acute challenge test, which may have underestimated the response to L-dopa in some cases. However, we reasoned that this relatively lower dose was appropriate, as it would be better tolerated by patients. Furthermore it is an approach that has been adopted in other similar observational studies [23], and in fact we found that the dose used in our challenge tests was not significantly different between definite and limited responders. However, the LEDD at the time of the challenge test was significantly lower (around 50mg per day) in patients with a limited response compared to those with a definite response, while there was no difference in LEDD between the groups at study entry. This raises the possibility that some patients are under-dosed despite worsening motor severity. Other factors may influence the drug dose given to some patients, such as neuropsychiatric features [24]. Our findings suggest that clinicians are more likely to increase dopamine replacement therapy doses when there is a stronger L-dopa response.

Earlier literature reported acute challenge tests with higher L-dopa doses. For example, a unit dose of $250 \mathrm{mg}$ L-dopa gave a positive response (i.e. a reduction in motor score equivalent to the threshold used in the present report) in 39 of 55 early PD cases (70.9\%) [8]. An earlier systematic review of dopaminergic challenge tests in PD, which included 2 studies using acute L-dopa challenges, calculated that $69 \%$ of de novo PD and 76\% of established PD exceeded this same threshold of response, but this combined results using $250 \mathrm{mg} \mathrm{L-dopa} \mathrm{in} 45$ cases, $200 \mathrm{mg} \mathrm{L}$-dopa in 1 case, and 100mg L-dopa in 21 cases [25]. Direct comparison with our findings is difficult, as those previous studies used a mixture of tasks and degrees of improvement to assess response (e.g. walking speed, tapping tasks, and 15-20\% improvement in motor scores) and only 1 of the 4 studies assessed the acute L-dopa challenge response using UPDRS 3 scores [25]. Assessing the effect of escalating L-dopa challenge test doses is the subject of few reports: in one study 16 out of 22 cases $(72.7 \%$ ) responded at $100 \mathrm{mg}$ L-dopa, which increased by 1 case (to $77.2 \%$ ) when the L-dopa challenge dose was increased to 200mg [26]. Further support of the impact of the L-dopa dose comes from the ELLDOPA study [3]. Considering those cases that had more than a 10\% improvement in motor score after 24 weeks of L-dopa treatment compared to baseline, this accounted for $76.5 \%$ of cases 
prescribed $300 \mathrm{mg}$ L-dopa per day, compared to $89 \%$ of cases prescribed $600 \mathrm{mg}$ Ldopa per day [2].

The other major consideration in L-dopa responsiveness relates to known intersubject variability in its pharmacokinetics. Variations in body weight [27], gut absorption [28] and gender [29] are all reported as potential contributors to response variations. The known higher bioavailability of L-dopa in postmenopausal women [29] did not, however, translate to a gender differences in our study. There are several other biological mechanisms that influence pharmacokinetics and pharmacodynamics of L-dopa at the cellular level. Higher levels of erythrocyte catechol-O-methyltransferase (which catabolises L-dopa) may impair the L-dopa response [30], and genetic variants of this [31] and other enzymes, including dopa decarboxylase [32] and monoamine oxidase type B [31], are emerging as contributors to later motor fluctuations associated with L-dopa. We consider it likely that genetic influences in the pharmacodynamic and pharmacokinetic handling of Ldopa will also influence the motor response to this drug even at earlier disease stages. The results of these detailed genetic analyses (including data about motor complications from our ongoing observations) will be the subject of a later report from our group.

\section{Acknowledgements}

The research was funded by Parkinson's UK and supported by the National Institute for Health Research (NIHR) DeNDRoN network, the NIHR Newcastle Biomedical Research Unit based at Newcastle upon Tyne Hospitals NHS Foundation Trust and Newcastle University, and the NIHR funded Biomedical Research Centre in Cambridge. The views expressed are those of the authors and not necessarily those of the NHS, the NIHR or the Department of Health. RAB is an NIHR Senior Investigator.

\section{Author contributions:}


NM: Data collection, analysis, manuscript writing and editing

MAL, SK, VP: Data analysis, manuscript writing and editing

NB, TF: Data collection, manuscript editing

JH, NMW, NW: Study design

DJB, RAB, HRM: Study design, data collection, manuscript editing

YBS: Study design, data analysis plan, manuscript editing

KAG, DGG: Study design, data collection, analysis, manuscript writing and editing

\section{Financial disclosures:}

N Malek, KA Grosset, MA Lawton, N Williams, Y Ben-Shlomo: No conflicts of interest

N Bajaj has received payment for advisory board attendance from UCB, Teva Lundbeck, Britannia, GSK, Boehringer, and honoraria from UCB Pharma, GE Healthcare, Lily Pharma, Medtronic, and BIAL Pharma.

He has received research grant support from GE Healthcare, Wellcome Trust, Medical Research Council, Parkinson's UK and National Institute for Health Research.

RA Barker has received grants from Parkinson's UK, NIHR, Cure Parkinson's Trust, Evelyn Trust, Rosetrees Trust, MRC, Wellcome Trust and EU along with payment for advisory board attendance from Oxford Biomedica and LCT, and honoraria from Wiley and Springer.

DJ Burn has received grants from Parkinson's UK and the Michael J Fox Foundation.

$\mathrm{J}$ Hardy has received honoraria from Eisai, and grant support from MRC/Wellcome, Parkinson's UK, and the Michael J Fox Foundation.

H R Morris has received grants from Medical Research Council UK, Wellcome Trust, Parkinson's UK, Ipsen Fund, Motor Neurone Disease Association, Welsh Assembly Government, PSP Association, CBD Solutions and Drake Foundation, and payment 
for advisory board attendance and lectures from Teva, AbbVie, Boehringer Ingelheim, and GSK.

DG Grosset has received honoraria from UCB Pharma, Acorda, and BIAL Pharma.

\section{References}

[1] K. Szewczyk-Krolikowski, P. Tomlinson, K. Nithi, R. Wade-Martins, K. Talbot, Y. BenShlomo, M.T. Hu, The influence of age and gender on motor and non-motor features of early Parkinson's disease: initial findings from the Oxford Parkinson Disease Center (OPDC) discovery cohort, Parkinsonism Relat Disord 20(1) (2014) 99-105.

[2] R.A. Hauser, P. Auinger, D. Oakes, Levodopa response in early Parkinson's disease, Mov Disord 24(16) (2009) 2328-36.

[3] S. Fahn, D. Oakes, I. Shoulson, K. Kieburtz, A. Rudolph, A. Lang, C.W. Olanow, C. Tanner, K. Marek, Levodopa and the progression of Parkinson's disease, N. Engl. J. Med. 351(24) (2004) 2498-508.

[4] A.J. Hughes, S.E. Daniel, L. Kilford, A.J. Lees, Accuracy of clinical diagnosis of idiopathic Parkinson's disease: a clinico-pathological study of 100 cases, J. Neurol. Neurosurg. Psychiatry 55(3) (1992) 181-4.

[5] R.B. Postuma, D. Berg, M. Stern, W. Poewe, C.W. Olanow, W. Oertel, J. Obeso, K. Marek, I. Litvan, A.E. Lang, G. Halliday, C.G. Goetz, T. Gasser, B. Dubois, P. Chan, B.R. Bloem, C.H. Adler, G. Deuschl, MDS clinical diagnostic criteria for Parkinson's disease, Mov Disord 30(12) (2015) 1591-601.

[6] A.J. Hughes, A.J. Lees, G.M. Stern, Challenge tests to predict the dopaminergic response in untreated Parkinson's disease, Neurology 41(11) (1991) 1723-5.

[7] M. Merello, E.R. Gerschcovich, D. Ballesteros, D. Cerquetti, Correlation between the Movement Disorders Society Unified Parkinson's Disease rating scale (MDS-UPDRS) and the Unified Parkinson's Disease rating scale (UPDRS) during L-dopa acute challenge, Parkinsonism Relat Disord 17(9) (2011) 705-7.

[8] M. Merello, M.I. Nouzeilles, G.P. Arce, R. Leiguarda, Accuracy of acute levodopa challenge for clinical prediction of sustained long-term levodopa response as a major criterion for idiopathic Parkinson's disease diagnosis, Mov Disord 17(4) (2002) 795-8. 
[9] C.G. Goetz, B.C. Tilley, S.R. Shaftman, G.T. Stebbins, S. Fahn, P. Martinez-Martin, W. Poewe, C. Sampaio, M.B. Stern, R. Dodel, B. Dubois, R. Holloway, J. Jankovic, J. Kulisevsky, A.E. Lang, A. Lees, S. Leurgans, P.A. LeWitt, D. Nyenhuis, C.W. Olanow, O. Rascol, A. Schrag, J.A. Teresi, J.J. van Hilten, N. LaPelle, Movement Disorder Societysponsored revision of the Unified Parkinson's Disease Rating Scale (MDS-UPDRS): scale presentation and clinimetric testing results, Mov. Disord. 23(15) (2008) 2129-70. [10] K.R. Chaudhuri, P. Martinez-Martin, R.G. Brown, K. Sethi, F. Stocchi, P. Odin, W. Ondo, K. Abe, G. Macphee, D. Macmahon, P. Barone, M. Rabey, A. Forbes, K. Breen, S. Tluk, Y. Naidu, W. Olanow, A.J. Williams, S. Thomas, D. Rye, Y. Tsuboi, A. Hand, A.H. Schapira, The metric properties of a novel non-motor symptoms scale for Parkinson's disease: Results from an international pilot study, Movement disorders : official journal of the Movement Disorder Society 22(13) (2007) 1901-11.

[11] K. Stiasny-Kolster, G. Mayer, S. Schafer, J.C. Moller, M. Heinzel-Gutenbrunner, W.H. Oertel, The REM sleep behavior disorder screening questionnaire--a new diagnostic instrument, Mov. Disord. 22(16) (2007) 2386-93.

[12] M. Visser, J. Marinus, A.M. Stiggelbout, J.J. Van Hilten, Assessment of autonomic dysfunction in Parkinson's disease: the SCOPA-AUT, Mov Disord 19(11) (2004) 1306-12. [13] G.T. Stebbins, C.G. Goetz, D.J. Burn, J. Jankovic, T.K. Khoo, B.C. Tilley, How to identify tremor dominant and postural instability/gait difficulty groups with the movement disorder society unified Parkinson's disease rating scale: comparison with the unified Parkinson's disease rating scale, Mov. Disord. 28(5) (2013) 668-70.

[14] M. Lawton, M.T. Hu, F. Baig, C. Ruffmann, E. Barron, D.M. Swallow, N. Malek, K.A. Grosset, N. Bajaj, R.A. Barker, N. Williams, D.J. Burn, T. Foltynie, H.R. Morris, N.W. Wood, M.T. May, D.G. Grosset, Y. Ben-Shlomo, Equating scores of the University of Pennsylvania Smell Identification Test and Sniffin' Sticks test in patients with Parkinson's disease, Parkinsonism \& related disorders 33 (2016) 96-101.

[15] R.P. Snaith, G.W. Bridge, M. Hamilton, The Leeds scales for the self-assessment of anxiety and depression, The British journal of psychiatry : the journal of mental science 128 (1976) 156-65.

[16] C.L. Tomlinson, R. Stowe, S. Patel, C. Rick, R. Gray, C.E. Clarke, Systematic review of levodopa dose equivalency reporting in Parkinson's disease, Mov Disord 25(15) (2010) 264953.

[17] N. Malek, M.A. Lawton, D.M. Swallow, K.A. Grosset, S.L. Marrinan, N. Bajaj, R.A. Barker, D.J. Burn, J. Hardy, H.R. Morris, N.M. Williams, N. Wood, Y. Ben-Shlomo, D.G. 
Grosset, Vascular disease and vascular risk factors in relation to motor features and cognition in early Parkinson's disease, Mov. Disord. 31(10) (2016) 1518-1526.

[18] J.A. Avina-Zubieta, H.K. Choi, M. Sadatsafavi, M. Etminan, J.M. Esdaile, D. Lacaille, Risk of cardiovascular mortality in patients with rheumatoid arthritis: a meta-analysis of observational studies, Arthritis Rheum. 59(12) (2008) 1690-7.

[19] M.M. Wickremaratchi, Y. Ben-Shlomo, H.R. Morris, The effect of onset age on the clinical features of Parkinson's disease, European journal of neurology : the official journal of the European Federation of Neurological Societies 16(4) (2009) 450-6.

[20] D.C. Velseboer, M. Broeders, B. Post, N. van Geloven, J.D. Speelman, B. Schmand, R.J. de Haan, R.M. de Bie, Prognostic factors of motor impairment, disability, and quality of life in newly diagnosed PD, Neurology 80(7) (2013) 627-33.

[21] F. Stocchi, C. Fossati, M. Torti, Rasagiline for the treatment of Parkinson's disease: an update, Expert opinion on pharmacotherapy 16(14) (2015) 2231-41.

[22] N. Malek, M.A. Lawton, K.A. Grosset, N. Bajaj, R.A. Barker, Y. Ben-Shlomo, D.J. Burn, T. Foltynie, J. Hardy, H.R. Morris, N.M. Williams, N. Wood, D.G. Grosset, Utility of the new Movement Disorder Society clinical diagnostic criteria for Parkinson's disease applied retrospectively in a large cohort study of recent onset cases, Parkinsonism \& related disorders 40 (2017) 40-46.

[23] The Parkinson Progression Marker Initiative (PPMI), Prog. Neurobiol. 95(4) (2011) 629-35.

[24] M. Lawton, Y. Ben-Shlomo, M.T. May, F. Baig, T.R. Barber, J.C. Klein, D.M.A. Swallow, N. Malek, K.A. Grosset, N. Bajaj, R.A. Barker, N. Williams, D.J. Burn, T. Foltynie, H.R. Morris, N.W. Wood, D.G. Grosset, M.T.M. Hu, Developing and validating Parkinson's disease subtypes and their motor and cognitive progression, Journal of Neurology, Neurosurgery \&amp; Psychiatry (2018).

[25] C.E. Clarke, P. Davies, Systematic review of acute levodopa and apomorphine challenge tests in the diagnosis of idiopathic Parkinson's disease, J Neurol Neurosurg Psychiatry 69(5) (2000) 590-4.

[26] D.F. D'Costa, L.J. Sheehan, P.A. Phillips, B. Moore-Smith, The levodopa test in Parkinson's disease, Age Ageing 24(3) (1995) 210-2.

[27] C. Warren Olanow, K. Kieburtz, O. Rascol, W. Poewe, A.H. Schapira, M. Emre, H. Nissinen, M. Leinonen, F. Stocchi, Factors predictive of the development of Levodopainduced dyskinesia and wearing-off in Parkinson's disease, Mov. Disord. 28(8) (2013) 106471. 
[28] A. Mukherjee, A. Biswas, S.K. Das, Gut dysfunction in Parkinson's disease, World journal of gastroenterology 22(25) (2016) 5742-52.

[29] P.A. LeWitt, Levodopa therapy for Parkinson's disease: Pharmacokinetics and pharmacodynamics, Mov Disord 30(1) (2015) 64-72.

[30] D.K. Reilly, L. Rivera-Calimlim, D. Van Dyke, Catechol-O-methyltransferase activity: a determinant of levodopa response, Clin. Pharmacol. Ther. 28(2) (1980) 278-86.

[31] T.F. Sampaio, E.U.D. Dos Santos, G.D.C. de Lima, R.S.G. Dos Anjos, R.C. da Silva, A.G.C. Asano, N.M.J. Asano, S. Crovella, P.R.E. de Souza, MAO-B and COMT Genetic Variations Associated With Levodopa Treatment Response in Patients With Parkinson's Disease, J. Clin. Pharmacol. 58(7) (2018) 920-926.

[32] D. Devos, S. Lejeune, F. Cormier-Dequaire, K. Tahiri, F. Charbonnier-Beaupel, N. Rouaix, A. Duhamel, B. Sablonniere, A.M. Bonnet, C. Bonnet, N. Zahr, J. Costentin, M. Vidailhet, J.C. Corvol, Dopa-decarboxylase gene polymorphisms affect the motor response to L-dopa in Parkinson's disease, Parkinsonism \& related disorders 20(2) (2014) 170-5.

\section{Figure Legends}

Figure 1. Patient recruitment to this study. The main analysis of L-dopa response was performed in all cases. Additional analysis was done in those cases without features that might be atypical for Parkinson's disease.

PD = Parkinson's disease $;$ MSA=Multiple System Atrophy; PSP = Progressive Supranuclear Palsy

Figure 2. MDS UPDRS 3 score at study entry and 18 months later, according to Ldopa response measured by challenge test, in 1007 patients with Parkinson's disease. Motor scores were significantly worse in those with a limited response at both time points. The increase was also significantly greater in those with a limited response, compared to those with a definite response (see text). Data are mean and standard deviation; datapoints are offset for clarity. 
MDS UPDRS = Movement Disorder Society Unified Parkinson's Disease Rating Scale.

Supplementary Figure 1. Frequency distribution of L-dopa response. The percentage improvement in MDS-UPDRS part 3 scores following L-dopa challenge dosing showed a bell-curve. The red vertical line indicates the $24.5 \%$ cut-off, above which the L-dopa response is considered excellent. 